\section{C'est un mystère qui}

nous accompagne depuis

des dizaines d'années :

la majeure partie de la matière de l'Univers (soit 83\%)

nous est invisible, et sa nature

complètement inconnue.

Au début on pensait qu'il

s'agissait d'astres éteints

ou de nuages de gaz

au rayonnement trop faible,

mais depuis 30 ans on sait

que cette matière n'est

pas ordinaire. Elle serait

au contraire composée

de particules entièrement

nouvelles, dont la nature

n’a jamais été dévoilée

ni dans l'espace ni dans

les accélérateurs de particules.

Ces particules sont traquées

dans les expériences

souterraines de détection

directe, mais toujours sans

succès. Si le neutralino,

le candidat favori provenant

des théories de supersymétrie,

n'est pas au rendez-vous dans

les prochaines années, quelles

seront les voies de sortie?

\title{
La matière noire, une sombre affaire
}

Françoise Combes (francoise.combes@obspm.fr)

Membre de l'Académie des sciences, professeure au Collège de France

LERMA (UMR 8112), Observatoire de Paris, 61 avenue de l'Observatoire, 75014 Paris

\section{Un peu d'histoire}

C'est dans les années 1930 que les premiers indices sont apparus clairement. Dès que l'astronome suisse Fritz Zwicky essaie de faire le bilan énergétique de l'amas de galaxies de Coma, il s'aperçoit du problème : l'énergie cinétique des galaxies est de plusieurs ordres de grandeur supérieure à leur énergie potentielle [1]. Pour les maintenir ensemble, il faudrait au moins 100 fois plus de masse que celle visible dans les galaxies. Peu à peu, ce résultat se généralise aux galaxies elles-mêmes. Les astronomes commencent à parler de matière manquante dans tout l'Univers et, à l'été 1961, une conférence se tient à Santa-Barbara en Californie pour discuter des solutions : naines noires, trous noirs, gaz ou grains solides d'hydrogène moléculaire, etc.

Puis dans les années 1970, les physiciens des particules s'en mêlent, et proposent des neutrinos, ou des gravitinos, ou autres cosminos issus des théories de supersymétrie pour rendre compte de la masse manquante [2]. Dans les années 1960-70, les courbes de rotation des galaxies spirales commencent à produire des résultats plus solides et détaillés : d'abord les premiers indices avec les mesures des vitesses Doppler en longueur d'onde visible, notamment par Vera Rubin et son équipe [3], montrent que les étoiles et le gaz ionisé au bord des galaxies tournent beaucoup plus vite que ne le laisse supposer leur masse visible. Puis les vitesses obtenues dans les longueurs d'onde radio avec le gaz d'hydrogène atomique, qui sonde la matière à plus grande distance du centre, montrent définitivement que toutes les galaxies sont entourées d'un halo de matière invisible $[4,5]$. Le terme "matière noire " (ou dark matter en anglais) commence à apparaître vers la fin des années 1970 [6], mais ne remplacera que très lentement le terme masse manquante, non lumineuse, ou masse invisible.
Jusqu'en 1980, pour la majorité des astronomes la matière noire devait être composée de matière ordinaire, donc de baryons. En 1984, il est définitivement établi que l'essentiel de la matière noire ne peut pas être de la matière baryonique, ceci pour deux raisons. La première est que l'abondance du deutérium et de l'hélium formés dans la nucléosynthèse primordiale n'est compatible avec les observations que si la fraction de baryons ne représente que 5\% de la densité critique (la densité nécessaire pour arrêter l'expansion de l'Univers par sa gravité). D'autre part, la formation des structures dans l'Univers sous l'effet de la gravitation est très lente à cause de l'expansion, et la matière ordinaire, ionisée et très couplée avec les photons, prend du retard pour s'effondrer sous l'effet de la gravité. Si l'on attend que la température dans l'expansion tombe en-dessous de 3000 degrés, pour que l'hydrogène se recombine et que le gaz s'effondre, il n'y a plus assez de temps pour former les galaxies. Il est donc nécessaire de disposer de matière exotique, sans interactions avec la lumière, qui peut s'effondrer tôt après le Big-Bang, avant même que la matière ordinaire puisse le faire [7]. Le nom de cette matière devrait alors être matière transparente, plutôt que matière noire : les rayons lumineux la traversent!

Il faut noter aussi que, dès les premières découvertes dans les années 1930, la solution "législative ", c'est-à-dire de changement des lois de la physique, a été avancée. Il est toutefois plus facile d'ajouter de la matière invisible à chaque fois qu'il en manque que d'imaginer et d'établir une théorie généralisant la loi de la gravité de Newton et la relativité générale. Une percée a été faite par le théoricien israélien Moti Milgrom dès 1983 [8], avec un modèle de gravité modifiée en champ faible (MOND) qui 


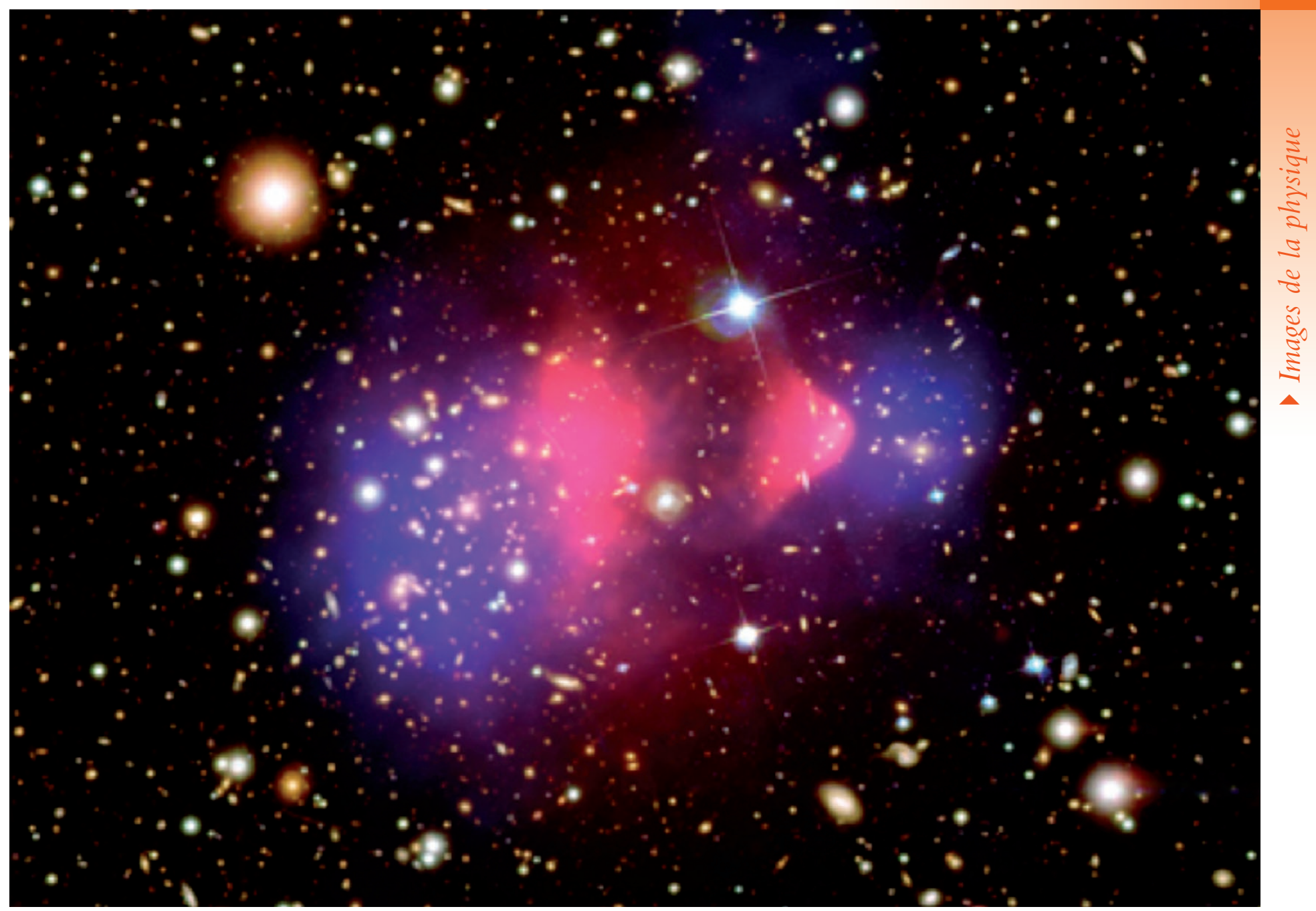

Collision entre deux sous-amas de l'amas de galaxies, dit « le Boulet ». Sur l'image optique du télescope spatial Hubble montrant les galaxies de l'amas en blanc sur fond noir, sont superposées les images en rayons X du gaz très chaud prises par le satellite Chandra (rose) et la carte de la masse totale déduite des lentilles gravitationnelles (bleu). Le gaz en rose montre nettement à droite une forme de cône, qui est l'onde de choc. L'angle du cône donne la vitesse du choc : Mach 3. (D’après Clowe et al. [12].)

rencontre beaucoup de succès à l'échelle des galaxies, mais des problèmes aux grandes échelles. La découverte de l'accélération de l'expansion de l'Univers et de l'énergie noire associée en 1998 (voir article p. 12) a relancé l'intérêt pour les théories modifiées qui, cette fois, seraient beaucoup plus rentables si elles permettaient de résoudre tout le secteur noir $70 \%$ d'énergie noire en plus de $25 \%$ de matière noire).

\section{Comment se distribue la matière noire?}

Aujourd'hui, les observations ont énormément progressé, et nous avons un recensement détaillé des régions de l'Univers où il manque de la masse, i.e. où la masse dynamique est supérieure à la masse visible. Au niveau des galaxies massives (voir l'encadré), la matière noire apparaît surtout dans leurs régions extérieures, comme un halo entourant le système

\section{Les différents types de galaxies}

Une galaxie est un ensemble d'étoiles, de gaz et de poussière, supposé entouré d'un halo de matière noire exotique. II y a plusieurs types de galaxies : spirales avec un disque d'étoiles et de gaz en rotation et un bulbe sphéroïdal au centre, ou elliptiques, avec seulement un sphéroïde d'étoiles vieilles. Les masses stellaires des galaxies varient de $10^{7} M_{\odot}$ (masses solaires) pour les galaxies naines, jusqu'à $10^{13} M_{\odot}$ pour les géantes (la masse stellaire de notre galaxie, la Voie lactée, est de l'ordre de $10^{11} \mathrm{M}_{\odot}$ ). La proportion de gaz et de matière noire est beaucoup plus élevée dans les galaxies naines. Les galaxies les plus massives sont elliptiques, sans gaz.

Les galaxies se forment peu après la naissance de l'Univers, lorsque du gaz tombe dans les halos de matière noire qui se sont effondrés auparavant, quand toute la matière ordinaire de I'Univers n'était encore que du gaz ionisé. Le gaz s'effondre lui-même pour former des étoiles, et la galaxie commence à briller.

Au cours de la vie d'une galaxie, du gaz s'écoule vers le centre et alimente un trou noir supermassif, de un million à un milliard de masses solaires. Il existe un trou noir supermassif au centre de chaque galaxie, de masse proportionnelle à la masse du bulbe. L'accrétion de gaz par le trou noir produit des phénomènes superlumineux et des vents relativistes qui peuvent éjecter le gaz environnant, et supprimer ainsi la formation d'étoiles. Ces phénomènes de rétroaction modèrent la croissance des galaxies. Une galaxie traversant un tel épisode est appelée galaxie à noyau actif. 


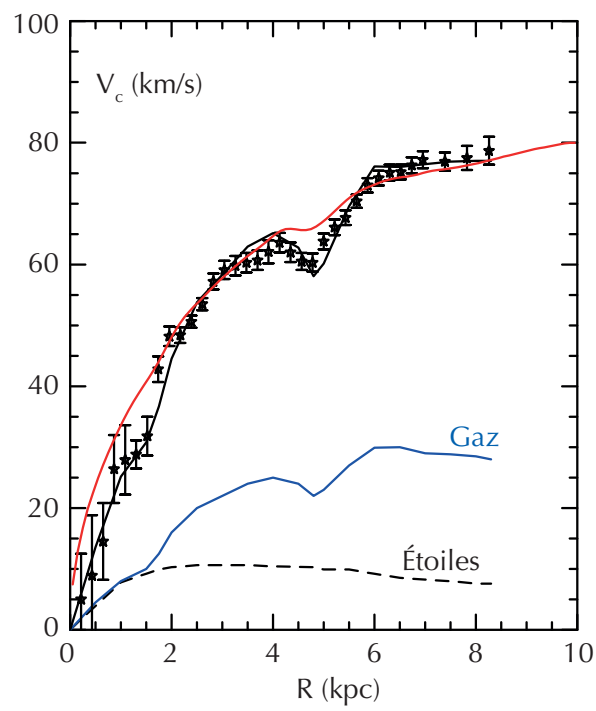

Galaxie géante - NGC 2903

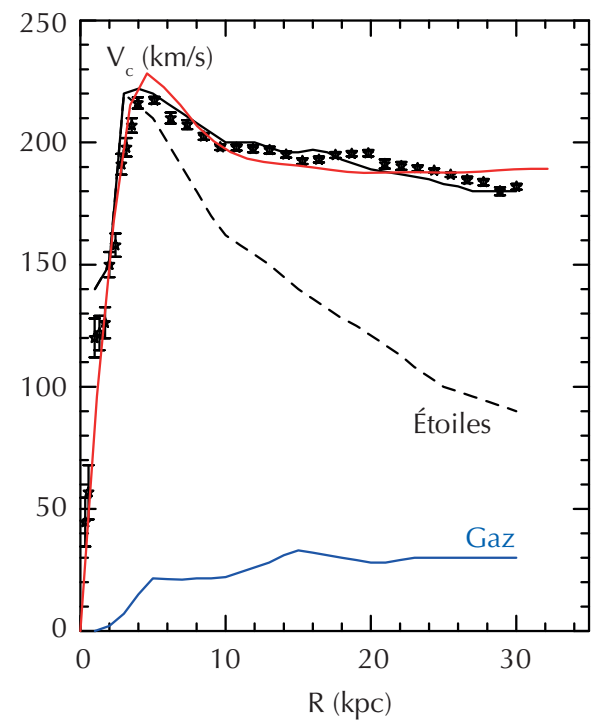

1. Vitesse de rotation $V_{c}$ mesurée à partir de la vitesse Doppler du gaz (symboles avec barres d'erreur) de deux galaxies en fonction de la distance $\mathbf{R}$ au centre (en kiloparsec, 1 pc $=3,26$ années-lumière). La courbe en tirets est la contribution des étoiles, et la courbe bleue la contribution du gaz au potentiel gravitationnel, et donc à la vitesse de rotation. Les courbes $V_{c}(R)$ observées tendent toutes (asymptotiquement pour les naines) vers un plateau, à grand rayon R. Dans la galaxie naine NGC 1560, la masse visible est dominée par le gaz, contrairement à la galaxie géante NGC 2903. Noter que la matière noire domine dès le centre dans la galaxie naine. L'ajustement des observations peut s'effectuer de deux façons : soit de la matière noire est introduite en supplément (courbe rouge), soit les baryons seuls sont considérés dans le cadre d'une théorie de la gravité modifiée (modèle MOND, courbe noire pleine).

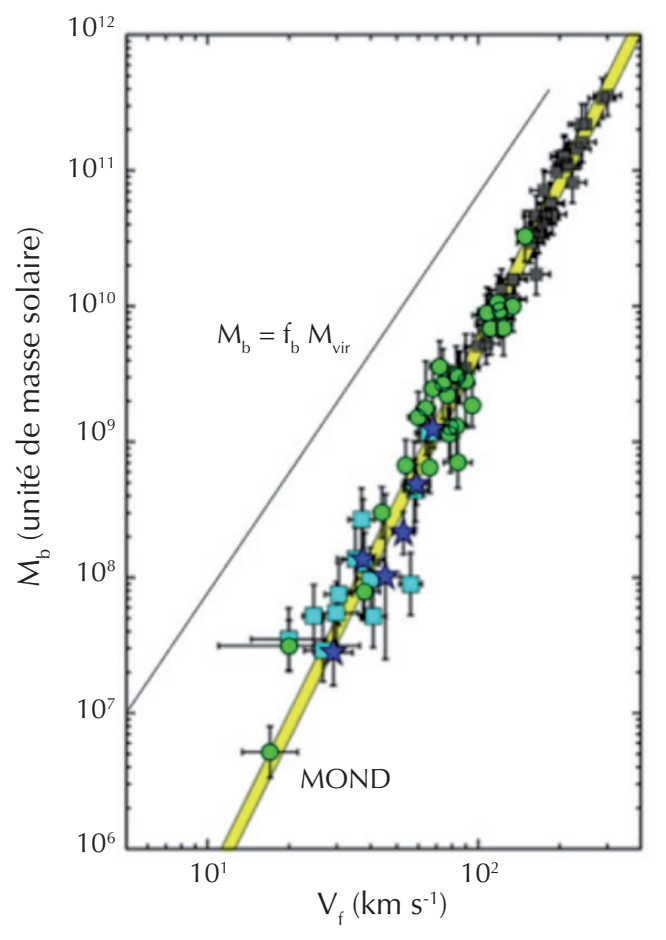

2. Lien entre la vitesse $V_{f}$ du plateau de la courbe de rotation et la masse de baryons $\mathbf{M}_{\mathbf{b}}$ dans les galaxies. L'axe horizontal est aussi un axe de masse : les galaxies naines sont à gauche et les géantes à droite. Les symboles bleus et verts sont les galaxies spirales dont la masse visible est dominée par le gaz, alors que les carrés gris correspondent aux galaxies dominées par les étoiles. La corrélation correspond à une loi de puissance de pente $4, \mathrm{M}_{\mathrm{b}} \sim \mathrm{V}^{4}$. C'est exactement ce qui est prédit par le modèle de gravité modifiée MOND (droite jaune), qui ne contient que des baryons. Dans le cas du modèle standard de matière noire froide, la courbe attendue est la droite noire fine, correspondant à une loi de puissance de pente 3 , si la fraction de baryons dans les galaxies est $f_{b}=17 \%$, la fraction de baryons globale dans l'Univers. Des processus de rétroaction dus aux supernovae ou aux noyaux actifs de galaxies sont invoqués pour éjecter les baryons des galaxies, et revenir sur la droite observée, tout en conservant le modèle standard de matière noire froide. (D'après McGaugh [11]).

\section{$\gg \gg$}

visible. Dans notre galaxie, la Voie lactée, le besoin de masse supplémentaire ne se fait sentir qu'au-delà de la position du Soleil, qui est situé à 25000 années-lumière du centre (soit la moitié du rayon du disque d'étoiles). L'étude détaillée des courbes de rotation de plus d'un millier de galaxies a montré que la proportion de matière invisible est d'autant plus grande que la densité surfacique du système d'étoiles est faible. Pour les galaxies massives, la densité par unité de surface des étoiles est trop élevée au centre pour nécessiter sans ambiguiité de la masse invisible. Par contre dans les galaxies naines, dont la densité surfacique au centre est de un ou plusieurs ordres de grandeur plus faible, la matière invisible domine dès le noyau (fig. 1).

Statistiquement, sur un très grand nombre de galaxies, il existe cependant une très bonne corrélation entre matière noire et matière visible. Depuis Tully \& Fisher [9] qui ont mis en évidence une relation très serrée entre vitesse de rotation des galaxies spirales, dépendant de leur halo de matière noire, et leur luminosité totale, de nombreuses versions de cette relation ont été proposées. Les plus précises relient la masse totale ordinaire (gaz et étoiles) à la vitesse du plateau de la courbe de rotation, obtenue à grande distance du centre (fig. 2).

Cette relation suggère que le contenu en matière ordinaire est un indicateur direct de la quantité de matière noire associée, au niveau des galaxies. En est-il de même à plus grande échelle?

Dans ce domaine, un outil s'est considérablement développé ces vingt dernières années : les lentilles gravitationnelles [10]. Une galaxie d'arrière-plan voit ses rayons lumineux déviés et son image déformée par une masse importante sur la ligne de visée, comme un amas de galaxies par exemple. En cas d'alignement entre galaxie de fond et lentille, il s'agit du phénomène de lentille forte : on observe de grands arcs gravitationnels (fig. 3), allant jusqu'à l'anneau d'Einstein. Mais en règle générale, les images de toutes les galaxies d'arrièreplan sont déformées, allongées de façon minime, et c'est l'ensemble statistique de ces centaines ou milliers de déformations qui renseigne sur la quantité de matière noire. C'est le phénomène de lentille faible, ou cisaillement gravitationnel, qui permet de cartographier la matière invisible. 


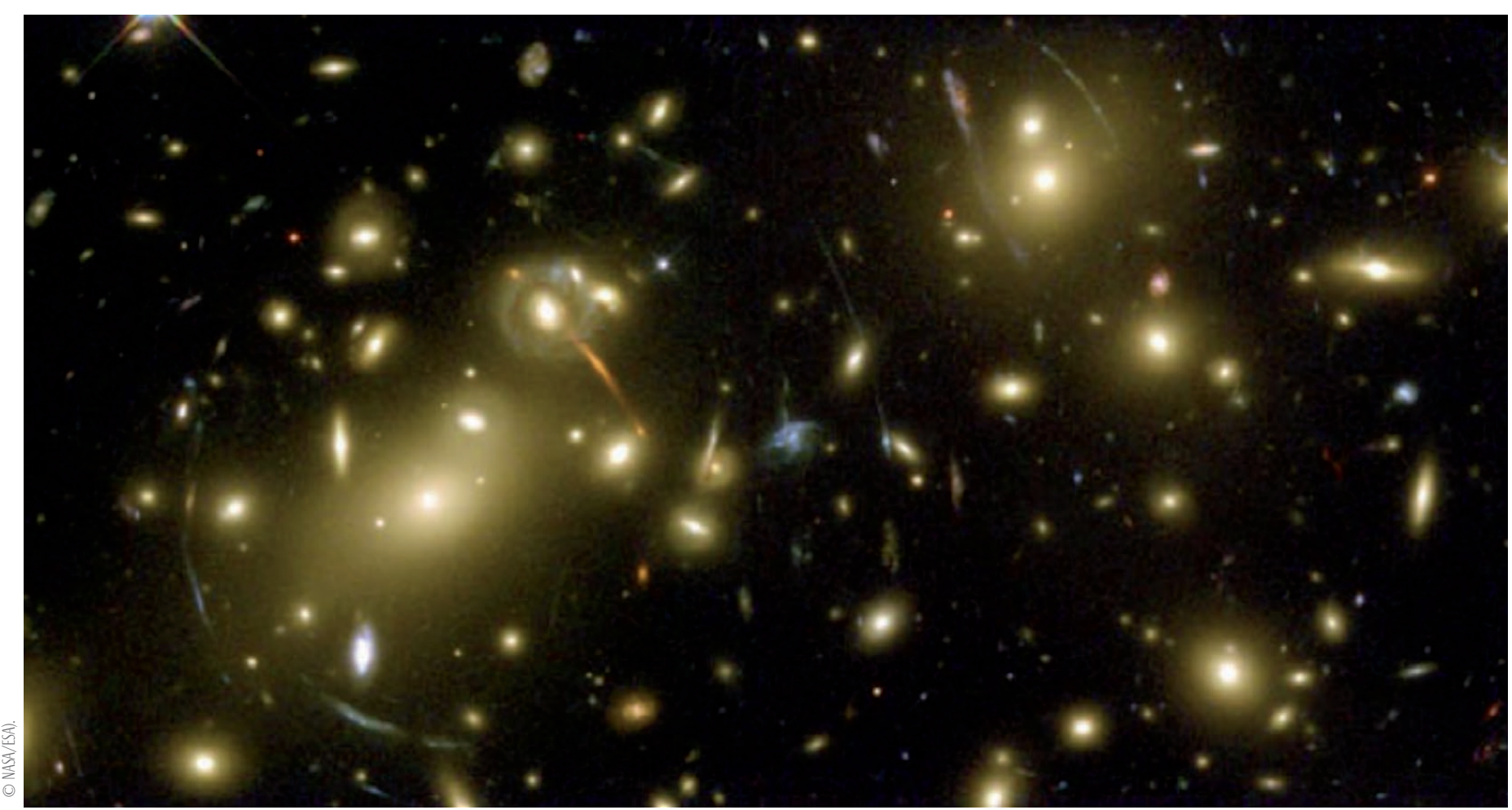

3. Exemple de lentilles gravitationnelles fortes dans l'amas de galaxies Abell 2218. Les différents arcs de lumière, de couleur variée, bleue ou orange, sont les images des galaxies d'arrière-plan déformées, car leurs rayons lumineux sont déviés par les fortes masses des galaxies de l'amas d'avant-plan. II existe aussi des milliers de petites galaxies de fond déformées seulement légèrement, et ce phénomène de lentilles faibles, ou cisaillement gravitationnel, permet statistiquement de cartographier la densité de surface de la matière noire sur la ligne de visée, dans tout l'amas. (Image du télescope Hubble.)

Les cartes de la matière noire obtenues ainsi, à l'échelle des amas de galaxies, correspondent dans la grande majorité des cas aux cartes de matière visible. Il existe pourtant des cas très intéressants de séparation spatiale des deux matières : les collisions violentes entre sous-amas. Le prototype de ces collisions est l'amas dit du Boulet (photo p. 5). Trois sortes de matière sont représentées ici : les galaxies qui se comportent comme un milieu sans collision, la matière noire cartographiée par les lentilles gravitationnelles, et un gaz très chaud, à des millions de degrés, qui émet des rayons $\mathrm{X}$. Le gaz est dissipatif, il est stoppé entre les deux amas par l'onde de choc de la collision. Or dans les amas riches de galaxies, le gaz chaud contient l'essentiel de la masse visible. La matière visible ne coïncide plus avec la matière noire! Celle-ci reste concentrée sur les galaxies. Cette séparation a permis de conclure à l'existence réelle de matière invisible, pouvant se déconnecter complètement de la matière ordinaire.

\section{Les différents modèles de matière noire}

Vers la fin des années 1970, les astronomes et physiciens soupçonnent que les neutrinos pourraient avoir une masse, contrairement à ce qui est prédit par le modèle standard des particules élémentaires. Si les trois espèces de neutrinos connus - électroniques, muoniques et tauiques - ont une masse, alors chaque neutrino peut se convertir en neutrino d'un autre type lors de sa propagation. L'existence de ces oscillations entre les trois types de neutrinos a permis de résoudre le mystère des neutrinos solaires (neutrinos électroniques), dont le flux observé est trois fois inférieur aux attentes. Plus tard, dans les années 1990, l'observation de ces oscillations pour les neutrinos atmosphériques a bien été confirmée [13]. Évidemment, dans les années 1980, les neutrinos ont été considérés comme candidats à la matière noire, vu leur abondance dans l'Univers, presque comparable à celle des photons. Il suffirait que leur masse soit le dix millionième de la masse de l'électron pour représenter toute la matière noire. Aujourd'hui la masse des neutrinos est estimée à 25 fois moins, et ne constituerait donc que $4 \%$ de la matière noire tout au plus. De toute façon, les simulations numériques des années 1980-90 montraient clairement que les neutrinos ne pouvaient pas constituer toute la matière noire, car faisant partie de la matière noire " chaude ". En effet, dès le changement de paradigme de 1984 sur la nature de la matière noire, les divers candidats peuvent être classés selon leur " température ". Le caractère " chaud " ou " froid " repose sur le critère de la vitesse moyenne qu'ont les particules lorsqu'elles se découplent du plasma primitif, où photons, baryons et matière noire sont en équilibre. Selon que les particules sont relativistes ou non lorsqu'elles se découplent de la soupe primitive juste après le Big-Bang, on parle de matière noire chaude (Hot Dark Matter ou HDM), ou de matière noire froide 

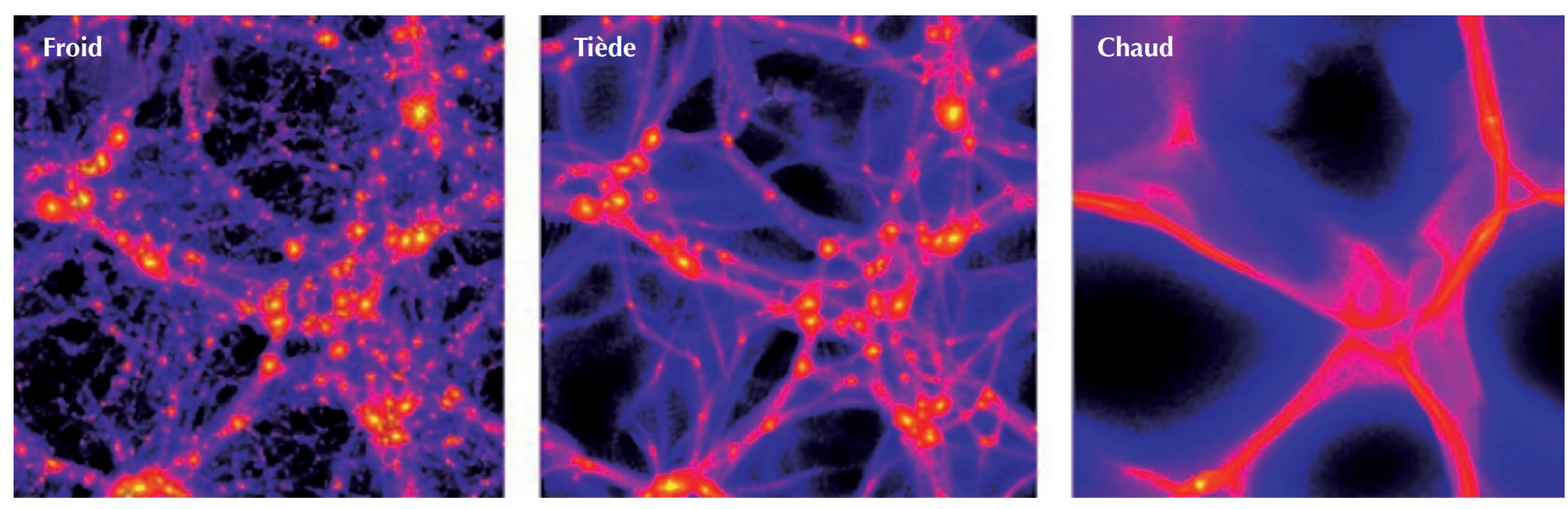

4. Simulations des grandes structures de l'Univers (échelle des amas et superamas de galaxies), dans le cadre des différents modèles de matière noire : froide (CDM), tiède (WDM) et chaude (HDM). Dans ce dernier modèle, les particules relativistes au début de l'Univers empêchent par leur pression l'effondrement de structures sur des échelles de la taille de l'horizon. Le modèle froid est celui qui représente le mieux les observations à grande échelle de l'Univers. La couleur représente la densité de matière noire : de noir-bleu pour les faibles densités, à jaune pour les plus fortes (les simulations ne considèrent que la matière noire).

$>>$

(Cold Dark Matter ou CDM). Les neutrinos font clairement partie de la première catégorie (HDM), même si aujourd'hui ils sont complètement ralentis et ne sont restés relativistes que durant les premiers 3-4\% de l'âge de l'Univers. La conséquence de leur vitesse relativiste est que ces particules trop chaudes vont empêcher les fluctuations de matière à petite échelle de se concentrer sous l'effet de leur propre gravité. En effet, les particules de matière noire chaude parcourent pratiquement les mêmes distances que les photons : elles ont un libre parcours moyen presque égal à l'horizon ${ }^{(a)}$, et leur " pression " stabilise la matière sur les échelles inférieures. Aux échelles supérieures, la matière pourra s'effondrer sous l'influence de sa propre gravité. Les structures de taille inférieure devront alors se former par fragmentation, dans un schéma top-down, du haut vers le bas.

Ce schéma ne correspond pas aux observations, notamment le spectre de masse des galaxies aux différents âges de l'Univers, qui favorisent le schéma contraire bottom-up, ou hiérarchique. Les premières structures à se former sont les plus petites structures instables, qui forment ensuite les grandes structures par fusions successives. Les simulations numériques de l'évolution de l'Univers avec les différents types de matière noire (fig. 4) ont montré que le modèle CDM, maintenant appelé modèle standard, est celui qui représente le mieux la formation des grandes structures et explique toutes les observations du fond cosmologique micro-onde, effectuées par les satellites WMAP et Planck : oscillations acoustiques et anisotropies du champ de photons, structure à grande échelle de la matière, oscillations acoustiques gelées dans la distribution des galaxies.

Pourtant, à l'échelle des galaxies, les prédictions du modèle CDM ne sont plus compatibles avec les observations. En particulier, dans ce modèle, la matière noire standard s'accumule au centre des galaxies, ce qui est contraire à ce que montrent les mesures de courbes de rotation (fig. 1). De plus, les simulations cosmologiques dans le modèle standard (CDM) prédisent l'existence de milliers de galaxies satellites autour des galaxies massives, milliers de galaxies qui ne sont pas observées. Des solutions ont été avancées à partir du constat que la physique de la matière ordinaire, et en particulier celle de la formation des étoiles à partir du gaz, dans les halos de matière noire est mal connue : la première vague de formation d'étoiles au centre des galaxies naines pourrait produire beaucoup de supernovae, et tant d'énergie que tout le gaz serait soufflé, ce qui stopperait la formation de ces galaxies. En effet, les galaxies se forment à partir de gaz qui se transforme en étoiles. La plupart des galaxies satellites resteraient noires ou invisibles. Mais ces hypothèses se heurtent à l'observation des galaxies naines sphéroïdales autour de la Voie lactée : bien qu'en petit nombre, le rapport du nombre d'étoiles comparé à leur matière noire est supérieur de plusieurs ordres de grandeur aux prédictions du modèle CDM.
De l'autre côté du spectre de masse des galaxies, pour stopper la formation des étoiles dans les galaxies massives, l'énergie des flambées d'étoiles est insuffisante. Il faut alors recourir à l'énergie fournie par le trou noir supermassif que l'on sait exister au centre de chaque galaxie. En principe, les phénomènes énergétiques accompagnant la croissance du trou noir massif sont assez puissants pour détruire la galaxie elle-même. Tout le problème est dans le couplage entre le disque de la galaxie et ce flot d'énergie localisée dans le noyau ou dans un jet qui s'échappe du noyau actif et de la galaxie.

L'importance de ces phénomènes de rétroaction de la part des supernovae ou des noyaux actifs pour modérer ou stopper la formation d'étoiles et éloigner la matière ordinaire des galaxies est bien quantifiée dans la figure 2. Si tous les baryons se trouvaient dans les galaxies, avec la même fraction $\mathrm{f}_{\mathrm{b}}=17 \%$ que dans tout l'Univers, les observations devraient se trouver sur la droite notée $M_{b}=f_{b} M_{v i r}$, où la masse visible $\left(\mathrm{M}_{\mathrm{b}}\right)$ est proportionnelle à la masse totale (masse virielle $\mathrm{M}_{\text {vir }}$ ) des galaxies, avec ce facteur universel $\mathrm{f}_{\mathrm{b}}$. Le fait que les observations s'alignent en dessous, d'un facteur 100 pour les galaxies naines, jusqu'à un facteur 3 pour les plus massives, nous indique que $99 \%$ des baryons doivent être éjectés des galaxies naines, et 2/3 des galaxies géantes. Cette éjection serait le fait des supernovae pour les galaxies de petite masse, et des noyaux actifs pour les galaxies massives. 
L'autre problème du modèle standard CDM est l'accumulation des particules de matière noire au centre des galaxies, en un pic de densité qui n'est pas observé. Pour le résoudre, il a été proposé un modèle de particules de matière noire qui entrent en collisions entre elles. Ces collisions agiraient surtout dans les parties les plus denses au centre, et auraient pour effet de disperser les particules et de rendre leurs trajectoires aléatoires dans cette région. Elles se distribueraient alors selon un nuage étendu, un " cœur ", au lieu d'un pic de matière noire. Il reste toutefois beaucoup de problèmes à résoudre pour expliquer la taille des cœurs différente d'une galaxie à l'autre. D'autre part, une limite supérieure de la section efficace de collisions entre particules de matière noire a été déduite des amas de galaxies en collision, comme le Boulet (photo p. 5).

Enfin, d'autres solutions sont explorées pour résoudre le problème des galaxies satellites manquantes : recourir à la matière noire "tiède" (fig. 4). Les structures à très petite échelle sont alors supprimées, mais une grande partie du modèle hiérarchique subsiste.

\section{Recherche expérimentale des particules candidates à la matière noire}

Il est remarquable que la masse des particules de matière noire ne soit pas connue à plusieurs ordres de grandeur près. On sait toutefois que ces particules n'interagissent que très faiblement entre elles, d'où leur nom de WIMP ("Weakly Interacting Massive Particle”).

Depuis 1985, juste après le changement de paradigme qui a fait de la matière noire une matière exotique et transparente, les astronomes et physiciens cherchent à détecter les particules qui la composent par leur interaction, même très faible, avec de la matière ordinaire. La matière noire devrait exister partout autour de nous, et les particules de matière noire traverser la Terre en étant parfois diffusées par un noyau d'atome. C'est cette interaction que les expériences de détection souterraine, situées à l'abri des rayons cosmiques parasites dans les tunnels du Fréjus ou du Grand Sasso par exemple, essaient d'identifier. L'expérience la plus performante aujourd'hui est située aux États-Unis (LUX, Large Underground Xenon experiment), avec un détecteur de $370 \mathrm{~kg}$ de xénon liquide. Les interactions éventuelles produisent des électrons et de la lumière, détectée par des tubes amplificateurs de lumière. Jusqu'à présent, seules des particules de fond (neutrons, matière ordinaire) ont été détectées, mais aucune particule de matière noire. La prochaine étape aura lieu dans le tunnel du Grand Sasso, où a été inaugurée, en novembre 2015, l'expérience Xenon1T, dont le détecteur est constitué de trois tonnes de xénon, objet d'une collaboration entre laboratoires américains et européens (dont des Français). Il a commencé à collecter des données au printemps 2016. Plusieurs autres expériences sont prévues dans les années futures, notamment LUX-Zeplin, qui contiendra 7 tonnes de xénon. La figure 5 représente l'état de l'art en fonction de la masse supposée du WIMP dans le domaine le plus probable. Les limites supérieures éliminent déjà une grande partie des valeurs possibles de la section efficace d'interaction entre les WIMPs et la matière ordinaire. Bientôt la limite du

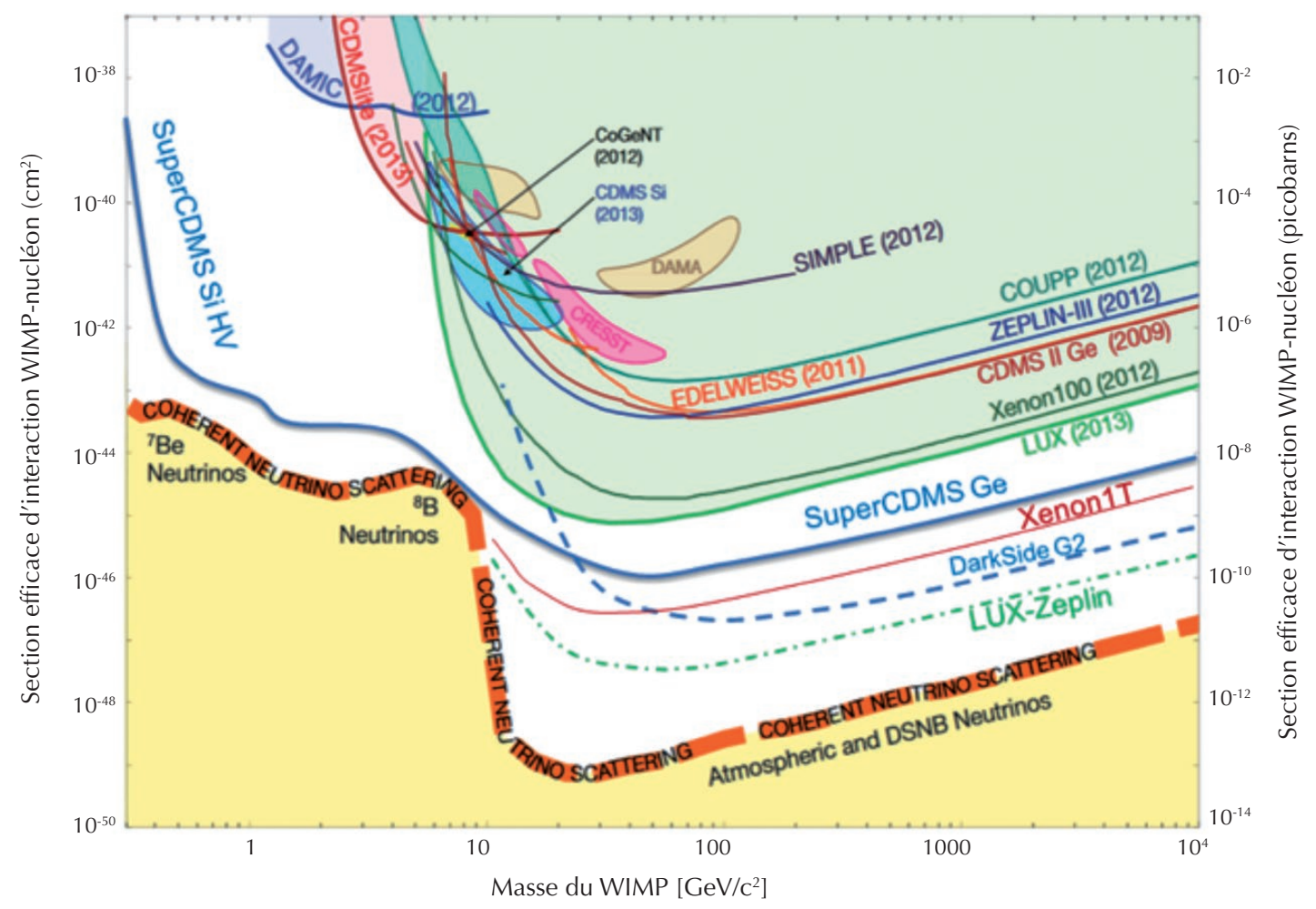

5. Limites supérieures pour la détection directe de particules de matière noire ou WIMPs, dans le diagramme masse - section efficace d'interaction avec les nucléons (matière ordinaire), en $\mathrm{cm}^{2}$ à gauche, ou picobarns à droite $\left(1 \mathrm{pb}=10^{-36} \mathrm{~cm}^{2}\right.$ ). Aujourd'hui, la meilleure limite est donnée par LUX (les zones colorées sont éliminées). Les expériences futures (SuperCDMS, Xenon1T, DarkSide G2, LUX-Zeplin) s'approchent du plancher des neutrinos (ligne orange), c'est-à-dire de la région du diagramme où les neutrinos provenant des supernovae, du Soleil ou de l’atmosphère, diffusés élastiquement par les nucléons, rendent la détection directe de WIMPs quasiment impossible. (Adapté de Billard et al. [15].) 
$>>$

fond de neutrinos sera atteinte, et la détection des WIMPs ne sera plus possible en raison de la confusion avec l'interaction des neutrinos et des nucléons.

Pendant toutes ces années, le principal candidat à la matière noire froide (CDM) a été le neutralino. C'est une particule hypothétique qui fait partie de l'extension au-delà du modèle standard des particules élémentaires, permise par la théorie de supersymétrie. Chaque particule connue aurait une particule supersymétrique partenaire, plus massive, et de spin complémentaire (i.e. un fermion pour un boson, et réciproquement). Le neutralino est la particule la plus stable de cet ensemble, dans lequel les autres particules supersymétriques se désintègrent. Le neutralino est une combinaison linéaire de trois particules : le zino, le photino et le Higgsino, superpartenaires de bosons (Z, photon et Higgs). Selon les facteurs de cette combinaison, on peut former quatre types de neutralinos stables. Pour une masse de l'ordre de 100 fois la masse du proton, une coïncidence encourageante favorise les neutralinos : ces particules WIMP sont leurs propres antiparticules et s'annihilent entre elles dans les premiers instants de l'Univers, avec une section efficace qui est celle de l'interaction nucléaire faible. Lorsque leur densité décroît avec l'expansion de l'Univers, si bien que le taux d'annihilation devient négligeable, leur abondance est gelée à une densité relique qui correspond bien à celle de la matière noire aujourd'hui. C'est le miracle du WIMP. Bien que le LHC n'ait pas mis en évidence ces particules jusqu'à présent, on peut encore espérer les découvrir à plus haute énergie.
Si l'annihilation est stoppée pratiquement dans tout l'Univers à cause de sa faible densité, il existe aujourd'hui quelques régions de surdensité où les annihilations pourraient reprendre, et les rayons gamma qui en résulteraient représentent un moyen de détection indirecte. Vers le centre de la Galaxie par exemple, les spectres des rayons gamma émis ont été étudiés en détail, mais ont toujours été expliqués par une source astrophysique : aucune détection indirecte de matière noire n'a pu être reportée de façon convaincante.

\section{Alternatives sans nouvelles particules}

Après trente ans de tentatives infructueuses de détection directe de particules de matière noire, et l'absence de détection de nouvelles particules au-delà du modèle standard au LHC du CERN, certains astronomes commencent à explorer de nouvelles pistes pour résoudre le mystère de la matière noire. Et si finalement il n'existait pas de matière inconnue, mais qu'une modification de la théorie de la gravité pouvait résoudre le problème de la masse manquante dans les galaxies et de la formation des structures de l'Univers ? À l'échelle des galaxies, le modèle de gravité modifiée qui représente le mieux les observations est celui de M. Milgrom, MOND, qui reproduit les courbes de rotation des naines aux géantes, de même que les relations d'échelle (fig. 1 et fig. 2). En champ de gravité faible, la force de gravité ne décroît alors plus avec la distance en $1 / R^{2}$, mais en $1 / R$. C'est dans le cadre de ce modèle qu'une théorie covariante relativiste a été construite par Bekenstein en 2004 [14], comprenant des champs tensoriel, vectoriel et scalaire $(\mathrm{TeVeS})$. Mais cette théorie a des difficultés pour les amas de galaxies et les structures à très grande échelle. De plus, la théorie relativiste n'est pas exempte d'instabilités internes.

Certains étudient les théories covariantes "Einstein æther", d'autres considèrent des dimensions supplémentaires à l'Univers, dans lesquelles la gravité pourrait se déployer, ce qui donnerait une masse apparente plus grande aux particules que nous connaissons. Certains n'hésitent pas non plus à combiner les avantages de l'existence de matière noire avec ceux d'une théorie de gravité modifiée. De cette floraison d'idées, va-t-il enfin émerger la théorie miraculeuse qui va nous faire sortir du noir ? I

(a) L'horizon est défini à chaque instant par la distance parcourue par la lumière pendant l'âge de l'Univers $t$, soit ct (c est la vitesse de la lumière dans le vide).

\section{Références}

• F. Zwicky, Astrophys. J. 86, (1937) 217.

2•H. Pagels et J.R. Primack, Phys. Rev. Lett. 48 (1982) 223.

3• V.C. Rubin et al., Astrophys. J. 225 (1978) L107.

4• M.S. Roberts, Astron. J. 74 (1969) 859.

5• A. Bosma, Astron. J. 86 (1981) 1825.

6• S.D.M. White et M.J. Rees, MNRAS 183 (1978) 341.

7• G.R. Blumenthal et al., Nature 311 (1984) 517

8• M. Milgrom, Astrophys. J. 270 (1983) 365.

9• R.B. Tully et J.R. Fisher, A\&A 54 (1977) 661.

10• Y. Mellier, Reflets de la Physique 1 (2006) 5.

11• S.S. McGaugh, Astron. J. 143 (2012) 40.

12• D. Clowe et al., Astrophys. J. 604 (2004) 596.

13• S. Lavignac et M. Zito, Reflets de la Physique 50 (2016) 4.

14• J.D. Bekenstein, Phys. Rev. D 70 (2004) 083509.

15• J. Billard et al., Phys. Rev. D 89 (2014) 023524. 